\title{
The CPR black hole with acceleration
}

\author{
Usman A. Gillani $^{1,2, \mathrm{a}}$, Khalid Saifullah ${ }^{1, \mathrm{~b}}{ }_{(\mathbb{D})}$ \\ ${ }^{1}$ Department of Mathematics, Quaid-i-Azam University, Islamabad, Pakistan \\ 2 NUSASH Department, National University of Technology, Islamabad, Pakistan
}

Received: 10 May 2021 / Accepted: 8 September 2021 / Published online: 22 September 2021

(C) The Author(s) 2021

\begin{abstract}
Different deformations and modifications have been proposed in the Kerr black hole solution. In the socalled non-Kerr metric a deformation function was proposed. This approach has been generalized to include two different deformation functions to obtain the CPR black hole (Cardoso et al. in Phys Rev D 89:064007, 2014). In this letter we develop the accelerating version of this spacetime and study its thermodynamics.
\end{abstract}

The theory of general relativity (GR) has been a subject of continuous tests and verifications since its discovery. Black holes are considered an ideal laboratory to perform different experiments in the strong gravitational field region. Kerr solution is expected to represent the astrophysical black holes in GR. By studying the properties of radiation which are produced due to the gas in the accretion disk, one can test the Kerr nature of these compact objects. The idea is to employ a framework similar to the parametrized post-Newtonian formalism [1] which has already been applied successfully to test GR in the regime of weak gravitational field. This framework is obtained by introducing some deviation parameters which help to measure deviations from the Kerr solution. These kinds of metrics must be able to describe the gravitational field that exists in the surrounding of compact objects, in any theory of gravitation, by making suitable choices of these parameters.

There exists a large number of spacetimes in literature that represent deformations and modifications of the Kerr black hole solution [2-9]. One of the well known and important deformed metrics is the so-called non-Kerr spacetime presented by Johannsen and Psaltsis [2] and it is also a widely studied example of the bottom-top approach of the Kerr hypothesis [2-4]. It consists of some free parameters besides the mass and spin of the black hole. These free parameters are used to measure deviation from the Kerr metric. This metric

\footnotetext{
a e-mail: usmangillani.qau@ gmail.com

b e-mail: ksaifullah@fas.harvard.edu (corresponding author)
}

presents vacuum solutions of some unknown field equations (which are different from Einstein's field equations) and are axisymmetric and asymptotically flat. The non-Kerr spacetime is regular for the single deformation parameter and it does not contain any unphysical properties above the event horizon and the maximum range of spin parameters can be considered to represent a black hole. It is also useful to test the no-hair theorem near the black holes that do not explicitly depend on the field equations. This metric is further extended to include more deviation functions and electric charge [6$11]$.

The non-Kerr metric introduced by Johannsen and Psaltis has gained a particular importance in the gravity community. Taking first a deformed Schwarzschild black hole (multiplying the components $g_{t t}$ and $g_{r r}$ of the Schwarzschild black hole by a function $(1+h)$, a non-Kerr black hole which has infinite number of deviation parameters was obtained by applying Newman-Janis algorithm. This approach was extended by Cardoso et al. [7] to construct a metric having two different deformation functions in the components $g_{t t}$ and $g_{r r}$ of the Schwarzschild black hole and is named as the CPR black hole. The line element of this black hole is given by

$$
\begin{aligned}
d s^{2}= & -\left(1+h^{t}\right)\left(1-\frac{2 M r}{\rho^{2}}+\frac{q^{2}}{\rho^{2}}\right) d t^{2} \\
& -2 a \sin ^{2} \theta\left[H-\left(1+h^{t}\right)\left(1-\frac{2 M r}{\rho^{2}}+\frac{q^{2}}{\rho^{2}}\right)\right] d t d \phi \\
& +\frac{\rho^{2}\left(1+h^{r}\right)}{\Delta+a^{2} \sin ^{2} \theta h^{r}} d r^{2}+\sin ^{2} \theta\left\{\rho^{2}+a^{2} \sin ^{2} \theta\right. \\
& \left.\times\left[2 H-\left(1+h^{t}\right)\left(1-\frac{2 M r}{\rho^{2}}+\frac{q^{2}}{\rho^{2}}\right)\right]\right\} d \phi^{2}+\rho^{2} d \theta^{2},
\end{aligned}
$$

where $H=\sqrt{\left(1+h^{t}\right)\left(1+h^{r}\right)}, \rho^{2}=r^{2}+a^{2} \cos ^{2} \theta, \Delta=$ $r^{2}+a^{2}-2 M r+q^{2}$ and $h^{i}(r, \theta)$ has the general expression 
$h^{i}(r, \theta)=\sum_{k=0}^{\infty}\left(\epsilon_{2 k}^{i}+\epsilon_{2 k+1}^{i} \frac{M r}{\rho^{2}}\right)\left(\frac{M^{2}}{\rho^{2}}\right)^{k}, \quad i=t, r$.

$M$ and $q$ represent the mass and charge of the black hole and $a=J / M$ is the rotation parameter, $J$ being the angular momentum. Taking $\epsilon_{k}^{t}=\epsilon_{k}^{r}$ for any value of $k$ yields the charged non-Kerr metric [8]. From these components we observe that $g_{t t}$ is only depending on $h^{t}$ and $g_{r r}$ contains the terms of $h^{r}$ only. But $g_{t \phi}$ and $g_{\phi \phi}$ are depending on both $h^{t}$ and $h^{r}$ which gives a complex expression. This CPR metric reduces to the non-Kerr when the deformations are set to equal values. Kerr black hole is obtained when we set deformations equal to zero.

The deformation parameters $\epsilon_{0}^{t}=\epsilon_{1}^{t}=\epsilon_{0}^{r}=\epsilon_{1}^{r}$ vanish when we require that the metric (1) is asymptotically flat. Here $\epsilon_{2}^{t}$ is constrained at the value $10^{-4}$ by parametrized post-Newtonian bound and hence is ignored, but there is no constraint on $\epsilon_{2}^{r}$. Therefore the deformation parameters start from $\epsilon_{3}^{t}$ and $\epsilon_{2}^{r}$. To match the results with the non-Kerr metric and its charged form in the limit $\epsilon_{3}^{r}=\epsilon_{3}^{t}$, we take $\epsilon_{3}^{r}$ and $\epsilon_{3}^{t}$ as the only nonzero parameters. For further study, they will be referred to as only $\epsilon^{r}$ and $\epsilon^{t}$ for convenience.

In this letter we construct an accelerating version of the CPR metric [7] and propose its two forms, one of them is electrically charged and the other is uncharged.

Plebański and Demiański introduced a spacetime in 1976 which covers a large family of electro-vacuum solutions in GR. The solution of accelerating and rotating black holes plays a vital role in this family of spacetimes. Here the parameter $\alpha$ measures the acceleration of the black hole. This metric represents the uniformly accelerating Kerr-type black holes. If the cosmological constant is taken to be zero, the charged accelerating black hole solution is [12-17]

$$
\begin{aligned}
d s^{2}= & \frac{1}{\Omega^{2}}\left\{-\left(\frac{Q}{\rho^{2}}-\frac{a^{2} P \sin ^{2} \theta}{\rho^{2}}\right) d t^{2}+\frac{\rho^{2}}{Q} d r^{2}+\frac{\rho^{2}}{P} d \theta^{2}\right. \\
& \left.+\sin ^{2} \theta\left(\frac{P\left(r^{2}+a^{2}\right)^{2}}{\rho^{2}}-\frac{Q a^{2} \sin ^{2} \theta}{\rho^{2}}\right) d \phi^{2}\right\} \\
& -\frac{2 a \sin ^{2} \theta\left[P\left(a^{2}+r^{2}\right)-Q\right]}{\rho^{2} \Omega^{2}} d t d \phi,
\end{aligned}
$$

where

$$
\begin{aligned}
\Omega & =1-\alpha r \cos \theta \\
\rho^{2} & =r^{2}+a^{2} \cos ^{2} \theta, \\
P & =1-2 \alpha M \cos \theta+\alpha^{2}\left(a^{2}+q^{2}\right) \cos ^{2} \theta, \\
Q & =\left(a^{2}+q^{2}-2 M r+r^{2}\right)\left(1-\alpha^{2} r^{2}\right) .
\end{aligned}
$$

Putting $\alpha=0$ gives the Kerr-Newman metric. Setting $\alpha=0=q$ yields the Kerr metric.
We propose the following CPR black hole spacetime which is accelerating as well:

$$
\begin{aligned}
d s^{2}= & \frac{1}{\Omega^{2}}\left\{-\left(\frac{Q}{\rho^{2}}-\frac{a^{2} P \sin ^{2} \theta}{\rho^{2}}\right)\left(1+h^{t}\right) d t^{2}\right. \\
& +\frac{\rho^{2}\left(1+h^{r}\right)}{Q+a^{2} h^{r} \sin ^{2} \theta} d r^{2}+\frac{\rho^{2}}{P} d \theta^{2} \\
& +\left[\left(\frac{P\left(r^{2}+a^{2}\right)^{2}}{\rho^{2}}-\frac{Q a^{2} \sin ^{2} \theta\left(1+h^{t}\right)}{\rho^{2}}\right) \sin ^{2} \theta\right. \\
& \left.\left.+a^{2} \sin ^{4} \theta\left(2(H-1)+\frac{a^{2} h^{t} \sin ^{2} \theta}{\rho^{2}}\right)\right] d \phi^{2}\right\} \\
& -\frac{2 a \sin ^{2} \theta\left[H-\left(1+h^{t}\right)\left\{1-\frac{P\left(r^{2}+a^{2}\right)-Q}{\rho^{2}}\right\}\right]}{\Omega^{2}} d \phi d t,
\end{aligned}
$$

where

$$
\begin{aligned}
\Omega & =1-\alpha r \cos \theta \\
\rho^{2} & =r^{2}+a^{2} \cos ^{2} \theta \\
P & =1-2 \alpha M \cos \theta+\alpha^{2}\left(a^{2}+q^{2}\right) \cos ^{2} \theta \\
Q & =\left(a^{2}+q^{2}-2 M r+r^{2}\right)\left(1-\alpha^{2} r^{2}\right) .
\end{aligned}
$$

When deformations vanish we recover the original spacetimes as expected. If we put $\alpha=0$ and $Q=0$, we obtain, respectively, the charged $\mathrm{CPR}$ and simple CPR black holes $[6,7]$. On the other hand, we get accelerating non-Kerr spacetime if we set $\epsilon^{t}=\epsilon^{r}$ and simple non-Kerr by putting $\alpha=0$ as well [2,9]. The vector potential $A_{\mu}$ of the accelerating CPR is defined as

$$
A_{\mu}=\left(-\frac{q r}{\rho^{2}}, \frac{\sqrt{1+h^{r}}}{\sqrt{1+h^{t}}} \frac{q r}{Q+a^{2} h^{r} \sin ^{2} \theta}, 0, \frac{a q r \sin ^{2} \theta}{\rho^{2}}\right) .
$$

The event horizon of the metric (8) is located at the solution of the equation $g^{r r}=0$ giving $Q+a^{2} h^{r} \sin ^{2} \theta=0$ which is depending only on the deformation function $h^{r}$. We denote it by $r_{+}$and it clearly depends on the mass, charge and spin of the black hole. The redshift surface of accelerating CPR black holes is obtained from $g^{t t}=0$ which has dependence only on $h^{t}$ and has no new contributions. The horizon and the redshift functions have similarity with the corresponding formulas for the accelerating non-Kerr black holes [9].

We study horizon thermodynamics of these objects here. The surface gravity $\kappa$ of a black hole is given as

$\kappa=\frac{1}{\sqrt{-h}} \frac{\partial}{\partial x^{a}}\left(\sqrt{-h} h^{a b} \frac{\partial r}{\partial x^{b}}\right)$, 
where $h^{a b}$ denotes the inverse of the metric deduced from the $t-r$ sector of the spacetime, $h=\operatorname{det} h_{a b}, \partial / \partial x^{a}$ denotes the partial derivative and the indices $a, b$ take the values 0,1 . Putting the values of $h^{11}$ and $\sqrt{-h}$ from Eq. (8), the surface gravity $\kappa_{+}$at the event horizon $r_{+}$, takes the form

$$
\begin{aligned}
\kappa_{+}= & \frac{\Omega^{2}}{2\left(1+h^{r}\right) \rho^{2}}\left\{\left(1-\alpha^{2} r_{+}^{2}\right)\left(2 r_{+}-2 M\right)\right. \\
& -2 \alpha^{2} r_{+}\left(a^{2}+q^{2}+r_{+}^{2}-2 M r_{+}\right) \\
& +a^{2} \epsilon^{r} M^{3} \sin ^{2} \theta\left[\frac{1}{\left(r_{+}^{2}+a^{2} \cos ^{2} \theta\right)^{2}}\right. \\
& \left.\left.-\frac{4 r_{+}^{2}}{\left(r_{+}^{2}+a^{2} \cos ^{2} \theta\right)^{3}}\right]\right\} .
\end{aligned}
$$

The Hawking temperature, $T=\kappa_{+} / 2 \pi$, at the event horizon can be obtained by taking the value of $\kappa_{+}$from the above equation as

$$
\begin{aligned}
T= & \frac{\Omega^{2}}{4 \pi\left(1+h^{r}\right) \rho^{2}}\left\{\left(1-\alpha^{2} r_{+}^{2}\right)\left(2 r_{+}-2 M\right)\right. \\
& -2 \alpha^{2} r_{+}\left(a^{2}+q^{2}+r_{+}^{2}-2 M r_{+}\right) \\
& +a^{2} \epsilon^{r} M^{3} \sin ^{2} \theta\left[\frac{1}{\left(r_{+}^{2}+a^{2} \cos ^{2} \theta\right)^{2}}\right. \\
& \left.\left.-\frac{4 r_{+}^{2}}{\left(r_{+}^{2}+a^{2} \cos ^{2} \theta\right)^{3}}\right]\right\} .
\end{aligned}
$$

To discuss entropy of the black holes, the first thing to be determined is the angular velocity $\omega$, which leads to the horizon area. We have

$\varphi_{+}=\frac{2 q r_{+}\left[H \rho^{2}-\left(1+h^{t}\right)\left\{\rho^{2}-P\left(a^{2}+r_{+}^{2}\right)-Q\right\}\right]}{a \sin ^{2} \theta\left[2 \rho^{2}(H-1)-Q\right]+P\left(a^{2}+r_{+}^{2}\right)^{2}+h^{t} a^{2} \sin ^{2} \theta\left[a^{2} \sin ^{2} \theta-Q\right]}$.

By substituting the values of $\kappa_{+}, \omega_{+}$and $\varphi_{+}$from Eqs. (15), (18) and (22) in (20), the first law takes the form

The horizon area of a rotating black hole is defined [18] by $A=4 \pi a / \omega_{+}$. Using Eq. (18) we obtain the entropy, $=A / 4$, of the black hole as

We note that $S$ increases with $M$ in accordance with the of conservation of mass as $[19,20]$

$d M=\frac{\kappa_{+}}{8 \pi} d A+\omega_{+} d J+\varphi_{+} d q$,

where $\varphi_{+}$denotes the electrostatic potential of the black hole

$\varphi_{+}=\frac{4 \pi q r_{+}}{A}$.

Substituting the area expression in the above equation, we get the electrostatic potential for the accelerating CPR black hole

Substituting the required metric components, the angular

$S=\frac{\pi}{2}\left(\frac{P\left(a^{2}+r_{+}^{2}\right)^{2}+a^{2} \sin ^{2} \theta\left[2 \rho^{2}(H-1)-Q\left(1+h^{t}\right)+a^{2} h^{t} \sin ^{2} \theta\right]}{H \rho^{2}-\left(1+h^{t}\right)\left\{\rho^{2}-P\left(a^{2}+r_{+}^{2}\right)-Q\right\}}\right)$.

second law of black hole thermodynamics. Now, the first

$$
\begin{aligned}
& d M=\frac{1}{8 \pi\left(1+h^{r}\right) \rho^{2}}\left\{\left(1-\alpha^{2} r_{+}^{2}\right)\left(2 r_{+}-2 M\right)-2 \alpha r_{+}\left(a^{2}+q^{2}+r_{+}^{2}-2 M r_{+}\right)\right. \\
& \left.+a^{2} \epsilon^{r} M \sin ^{2} \theta\left[\frac{1}{\left(r_{+}^{2}+a^{2} \cos ^{2} \theta\right)^{2}}-\frac{4 r_{+}^{2}}{\left(r_{+}^{2}+a^{2} \cos ^{2} \theta\right)^{3}}\right]\right\} d A \\
& +\frac{2 a\left[H \rho^{2}-\left(1+h^{t}\right)\left\{\rho^{2}-P\left(a^{2}+r_{+}^{2}\right)-Q\right\}\right]}{P\left(a^{2}+r_{+}^{2}\right)^{2}+a^{2} \sin ^{2} \theta\left[2 \rho^{2}(H-1)-Q\left(1+h^{t}\right)+a^{2} h^{t} \sin ^{2} \theta\right]} d J \\
& +\frac{2 q r_{+}\left[H \rho^{2}-\left(1+h^{t}\right)\left\{\rho^{2}-P\left(a^{2}+r_{+}^{2}\right)-Q\right\}\right]}{a \sin ^{2} \theta\left[2 \rho^{2}(H-1)-Q\right]+P\left(a^{2}+r_{+}^{2}\right)^{2}+h^{t} a^{2} \sin ^{2} \theta\left[a^{2} \sin ^{2} \theta-Q\right]} d q .
\end{aligned}
$$

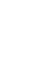


If we put $q=0$ in (8) we obtain the spacetime for the uncharged accelerating CPR black hole. The values of $P$ and $Q$ in this case become

$$
\begin{aligned}
& P=1-2 \alpha M \cos \theta+\alpha^{2} a^{2} \cos ^{2} \theta, \\
& Q=\left(a^{2}-2 M r+r^{2}\right)\left(1-\alpha^{2} r^{2}\right) .
\end{aligned}
$$

The surface gravity and the Hawking temperature at $r_{+}$will reduce to

$$
\begin{aligned}
& \kappa_{+}=\frac{\Omega^{2}}{2\left(1+h^{r}\right) \rho^{2}}\left\{\left(1-\alpha^{2} r_{+}^{2}\right)\left(2 r_{+}-2 M\right)\right. \\
& -2 \alpha^{2} r_{+}\left(a^{2}+r_{+}^{2}-2 M r_{+}\right) \\
& d M=\frac{1}{8 \pi\left(1+h^{r}\right) \rho^{2}}\left\{\left(1-\alpha^{2} r_{+}^{2}\right)\left(2 r_{+}-2 M\right)-2 \alpha r_{+}\left(a^{2}+e^{2}+r_{+}^{2}-2 M r_{+}\right)\right. \\
& \left.+a^{2} \epsilon^{r} M \sin ^{2} \theta\left[\frac{1}{\left(r_{+}^{2}+a^{2} \cos ^{2} \theta\right)^{2}}-\frac{4 r_{+}^{2}}{\left(r_{+}^{2}+a^{2} \cos ^{2} \theta\right)^{3}}\right]\right\} d A \\
& +\frac{2 a\left[H \rho^{2}-\left(1+h^{t}\right)\left\{\rho^{2}-P\left(a^{2}+r_{+}^{2}\right)-Q\right\}\right]}{P\left(a^{2}+r_{+}^{2}\right)^{2}+a^{2} \sin ^{2} \theta\left[2 \rho^{2}(H-1)-Q\left(1+h^{t}\right)+a^{2} h^{t} \sin ^{2} \theta\right]} d J .
\end{aligned}
$$

Table 1 Entropy for the accelerating CPR black hole, for the charged and uncharged cases, for some values of mass $M$

\begin{tabular}{lll}
\hline$M$ & $S$ (charged case) & $S$ (uncharged case) \\
\hline 50 & $14,810.78637$ & $14,811.17421$ \\
55 & $17,898.67605$ & $17,899.47966$ \\
60 & $21,301.05788$ & $21,301.86151$ \\
65 & $24,999.33684$ & $25,000.14047$ \\
70 & $28,993.48780$ & $28,994.29143$ \\
\hline
\end{tabular}

By substituting the above values, the first law for the uncharged black hole takes the form

$$
\begin{aligned}
& +a^{2} \epsilon^{r} M^{3} \sin ^{2} \theta \\
& \left.\times\left[\frac{1}{\left(r_{+}^{2}+a^{2} \cos ^{2} \theta\right)^{2}}-\frac{4 r_{+}^{2}}{\left(r_{+}^{2}+a^{2} \cos ^{2} \theta\right)^{3}}\right]\right\} \\
T= & \frac{\Omega^{2}}{4 \pi\left(1+h^{r}\right) \rho^{2}}\left\{\left(1-\alpha^{2} r_{+}^{2}\right)\left(2 r_{+}-2 M\right)\right. \\
& -2 \alpha^{2} r_{+}\left(a^{2}+r_{+}^{2}-2 M r_{+}\right) \\
& +a^{2} \epsilon^{r} M^{3} \sin ^{2} \theta \\
& \left.\times\left[\frac{1}{\left(r_{+}^{2}+a^{2} \cos ^{2} \theta\right)^{2}}-\frac{4 r_{+}^{2}}{\left(r_{+}^{2}+a^{2} \cos ^{2} \theta\right)^{3}}\right]\right\} .
\end{aligned}
$$

It can easily be seen that entropy in this case becomes
To conclude, we have formulated two deformed CPR black hole spacetimes with acceleration and studied the effects of the deviation parameters $\epsilon^{r}, \epsilon^{t}$ along with acceleration parameter $\alpha$, on the structure and thermodynamics of these holes. The well-known accelerating black hole solution and its thermodynamical properties are the limiting case (when the deformation parameters vanish) of these new metrics. Further, taking $\alpha=0$, all the results of Kerr black hole can be obtained.

The study of thermodynamics reveals that the black hole cannot be represented for the large values of acceleration parameter because the Hawking temperature becomes negative which violates the third law of thermodynamics. Only a small range of acceleration parameter provides the physically significant results $[21,22]$.

$S=\frac{\pi}{2}\left(\frac{P\left(a^{2}+r_{+}^{2}\right)^{2}+a^{2} \sin ^{2} \theta\left[2 \rho^{2}(H-1)-Q\left(1+h^{t}\right)+a^{2} h^{t} \sin ^{2} \theta\right]}{H \rho^{2}-\left(1+h^{t}\right)\left\{\rho^{2}-P\left(a^{2}+r_{+}^{2}\right)-Q\right\}}\right)$.

The entropy behaviour is further elaborated in Table 1. It is clear from here that the presence of charge has led to a decrease in the entropy.
The behaviour of the entropy has increasing values for both the spacetimes of accelerating CPR black holes and thus satisfy the condition of the second law of thermodynam- 
ics. This study can be extended by considering some other parameters like NUT parameters and cosmological constant along with the acceleration parameter $\alpha$ to analyze the effect of these deformations similar to the one presented in this study. It would be interesting to investigate particle dynamics and energy processes in the accelerating CPR spacetime presented here.

Acknowledgements Useful discussions with Rehana Rahim are gratefully acknowledged.

Data Availability Statement This manuscript has no associated data or the data will not be deposited. [Authors' comment: There is no data for depositing.]

Open Access This article is licensed under a Creative Commons Attribution 4.0 International License, which permits use, sharing, adaptation, distribution and reproduction in any medium or format, as long as you give appropriate credit to the original author(s) and the source, provide a link to the Creative Commons licence, and indicate if changes were made. The images or other third party material in this article are included in the article's Creative Commons licence, unless indicated otherwise in a credit line to the material. If material is not included in the article's Creative Commons licence and your intended use is not permitted by statutory regulation or exceeds the permitted use, you will need to obtain permission directly from the copyright holder. To view a copy of this licence, visit http://creativecomm ons.org/licenses/by/4.0/.

Funded by SCOAP ${ }^{3}$.

\section{References}

1. C.M. Will, Living Rev. Relativ. 9, 3 (2006)

2. T. Johannsen, D. Psaltis, Phys. Rev. D 83, 124015 (2011)

3. T. Johannsen, Phys. Rev. D 88, 044002 (2013)

4. K. Glampedakis, S. Babak, Class. Quantum Gravity 23, 4167 (2006)

5. R. Konoplya, L. Rezzolla, A. Zhidenko, Phys. Rev. D 93, 064015 (2016)

6. R. Rahim, K. Saifullah, The charged CPR black hole (submitted for publication)

7. V. Cardoso, P. Pani, J. Rico, Phys. Rev. D 89, 064007 (2014)

8. R. Rahim, K. Saifullah, Ann. Phys. 405, 220 (2019)

9. U.A. Gillani, R. Rahim, K. Saifullah, The non-Kerr black hole with acceleration. arXiv:2106.02095 [gr-qc]

10. F. Filippini, G. Tasinato, JCAP 01, 033 (2018)

11. S. Chen, J. Jing, Phys. Rev. D 89, 104014 (2014)

12. J.F. Plebański, M. Demiański, Ann. Phys. 98, 98 (1976)

13. J. Podolský, H. Kadlecový, Class. Quantum Gravity 26, 105007 (2009)

14. J.B. Griffiths, J. Podolský, Class. Quantum Gravity 22, 3467 (2005)

15. U.A. Gillani, K. Saifullah, Phys. Lett. B 699, 15 (2011)

16. M. Rehman, K. Saifullah, JCAP 1103, 01 (2011)

17. L. Vanzo, G. Acquaviva, R. Di Criscienzo, Class. Quantum Gravity 28, 183001 (2011)

18. V.P. Frolov, I.D. Novikov, Black Hole Physics: Basic Concepts and New Developments (Kluwer Academic Publishers, Dordrecht, 1998)

19. M. Akbar, K. Saifullah, Eur. Phys. J. C 67, 205 (2010)

20. M. Akbar, K. Saifullah, Gen. Relativ. Gravit. 43, 933 (2011)

21. M. Bilal, K. Saifullah, Astrophys. Space Sci. 343, 165 (2013)

22. O.J.C. Dias, J.P.S. Lemos, Phys. Rev. D 67, 084018 (2003) 